\title{
MUEGANO: A divide and conquer algorithm to overcome memory limitations when assembling shotgun projects
}

\author{
Octavio Martínez de la Vega ${ }^{1}$ and \\ Araceli Fernández Cortés ${ }^{1}$
}

\section{Abstract}

When assembling large quantity of reads in a genomic shotgun project a serious limitation is the amount of random access memory (RAM) of the computers used in the project. This arises because all assembling programs must look at all the overlaps between reads at the same time, using RAM in order to construct contigs, and the memory of the computer can be filled up during this step, causing the abortion of the assembling process.

Here we propose an algorithm that is capable of overcoming any memory limitation by using redundancy of processing and thus producing an increase in computing time but overcoming the memory limitation.

The proposed algorithm consists in dividing the reads in a set of groups which size is half the maximum capability in memory of the computer used and performing assembling for all the possible combination pairs of such groups. After eliminating the redundancy of the set of contigs obtained in the previous step, the process is iterated until a set of contigs of manageable size is obtained such that the set can be handled by the assembler in a final step.

Each step of the procedure increases the time of computing from $k$ to approximately $\mathrm{k}+\mathrm{k}(\mathrm{k}-1) / 2$, but in many practical cases only one step is needed to finish the assembling process. The procedure is suitable for any kind of assembler and was successfully applied to the assembly of a very large set of reads from the maize genome.

\section{Results}

The algorithm proposed here, called MUEGANO ${ }^{2}$ is capable of overcoming any memory limitation by using redundancy of processing and thus producing an increase in computing time. Even with an increase in computational time, this process could be the only practical way to process a very large number of reads in a shotgun assembling project that surpass the memory capacity of a given computer. The algorithm is warranted to recover all contigs formed by two or more reads.

The algorithm is carried out in steps that can be repeated until finishing the assembly, say:

1 - A set of redundant groups of reads of the maximum size permitted by the amount of RAM available are obtained and assembled producing a first order set of redundant contigs (contigs1).

\footnotetext{
${ }^{1}$ Laboratorio Nacional de Genómica para la Biodiversidad (Langebio), CINVESTAV Irapuato.

Km 9.6 Libramiento Norte Carretera Irapuato-León, 36500 Irapuato México.

${ }^{2}$ From Multiple Unrelated Element Genome Assembler in a Non-random Order.
} 
2 - A filtering procedure is applied to the set contigs1 to eliminate the redundancy, obtaining a set of filtered first order non-redundant contigs (Fcontigs1).

3 - If the number of contigs in Fcontigs 1 is small enough to be processed by a single run of the assembler then we proceed to step 4, otherwise step 1 is repeated using as reads the sequences of the set Fcontigs1.

4 - The set of non redundant contigs produced is feed to the assembler producing a final assembly.

Assume that the maximum number of reads that can be processed in one run by the assembler program is no more than $2 \mathrm{n}$ and also that we have a total of $\mathrm{N}$ reads to process, $\mathrm{N}>>\mathrm{n}$. Let $\mathrm{k}$ be the largest natural number that fulfils condition $k \geq N / n$; that is, the number of groups of reads of size $n$ that can be formed. To be precise let say that we number the reads from 1 to $\mathrm{N}$ and denote them by $r_{i}$ from $i=1$ to $\mathrm{N}$. Consider the $\mathrm{k}$ groups of $\mathrm{n}$ distinct reads denoted by $G_{j}, j=1,2, \ldots k$ where $G_{1}$ is formed by the first $n$ reads, $G_{2}$ by the following $n$ reads and so on until $G_{k}$ that contain the last $n$ reads, or less than $n$ reads if $k>$ $\mathrm{N} / \mathrm{n}$. Now consider forming all the possible combinations of two groups, say $E_{\mathrm{mn}}$ $=\left\{G_{m}, G_{n}\right\}$, where $m<n ; m=1,2, \ldots k-1, n=2,3, \ldots k$. Clearly there are $k(k-$ 1)/2 of such groups, because the first component of $E_{m n}$ can be selected from any of the $k$ groups and the second member can be selected from the remaining (k-1) and we divide by 2 because only sets with different sub index are desired. Each one of these $E_{m n}$ groups can be assembled in one run of the assembler given that they have at most $2 \mathrm{n}$ reads (see Figure 1 ).

The set of contigs obtained from running the assembler on the groups $\left\{\mathrm{E}_{\mathrm{mn}}\right\}$, say contig1, will contain all possible contigs of at least two reads and will also be redundant, because there are (multiple) cases where the same contig will be obtained in more than one group (see Proposition 1). We can filter this set to obtain a non-redundant set of contigs, Fcontig1. This can be done, for example, by using the blastclust program of the BLAST suit, coupled with a simple ad-hoc script.

\section{Original set of reads}
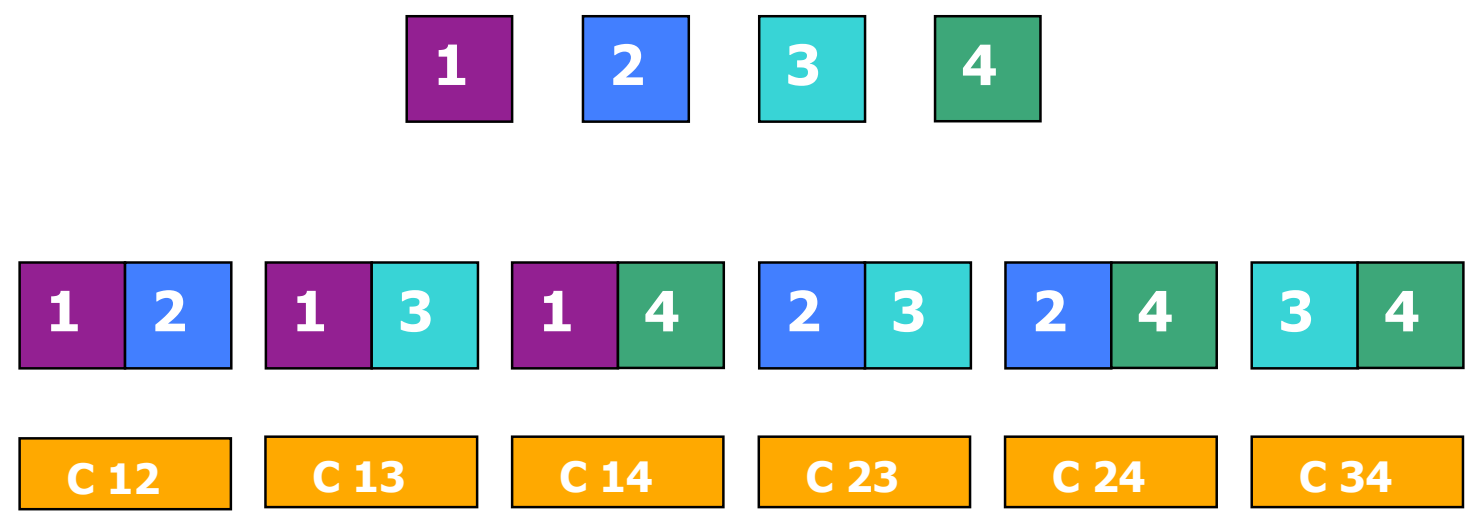

Figure 1. Example of the first step of MUEGANO. The total number of reads can be divided in four groups, and each possible assembly $(1,2),(1,3), \ldots(3,4)$ is performed giving the sets of (redundant) contigs c12, c13,... c34. 
All contigs obtained (Fcontig1) can be used as if they were simple reads to continue the assembling process, obtaining contigs 2 and Fcontigs2, etc. If in any step the number of contigs is less than $2 n$ they can be feed to the assembler to conclude the process; otherwise the procedure continues until the size of Fcontigs-t is of size $2 \mathrm{n}$ or less and can then be processed in one single run of the assembler producing the final set of contigs in step $t+1$. Only the "singletons" obtained in the first cycle will be real singletons (formed by one read); "singletons" obtained in the subsequent cycles will be in fact contigs formed by more than one read that did not increased in number of reads and will not increase in any subsequent cycle because no other read (singleton or contig) overlaps with it.

This procedure guarantees that all contigs will be recovered (see propositions 2 and 3 ).

If in a given step, say $u$, no new contigs are obtained in any of the sets (say that Fcontigs-u is empty) the procedure stops because there are not new significant overlaps to take into account.

In all propositions below it is assumed that the assembler program is perfect, that is, if an overlap of a given size $s$ exist between any two reads $x$ and $y$ and if such reads are feed to the assembler it will recover the contig formed by such reads, say $c_{x y}$.

\section{Proposition 1}

All contigs of exactly two reads will be present in at least one of the $E_{m n}$ assemblies.

Proof

Take any two reads, say read $x$ and read $y$, that can be assembled into a contig $c_{x y}$ because they overlap in a significant part. We need to see that contig $c_{x y}$ will be present in at least one of the $E_{m n}$ assemblies.

There are two possible cases, say: 1) Reads $x$ and $y$ are both in one of the groups, say $G_{m}$, or 2) Reads $x$ and $y$ are in two different groups, say that $x$ is in group $G_{m}$, and $y$ is in group $G_{n}$. In case one the contig $c_{x y}$ will be present in the result of assembling all $E_{m r}$ groups; $r=m+1, m+2, \ldots k$. In the second case the contig $C_{x y}$ will be present only in assembly $E_{m n}$. Given that not constrain was imposed in the selection of $x$ and $y$ this proves the proposition. (See Figure 2).

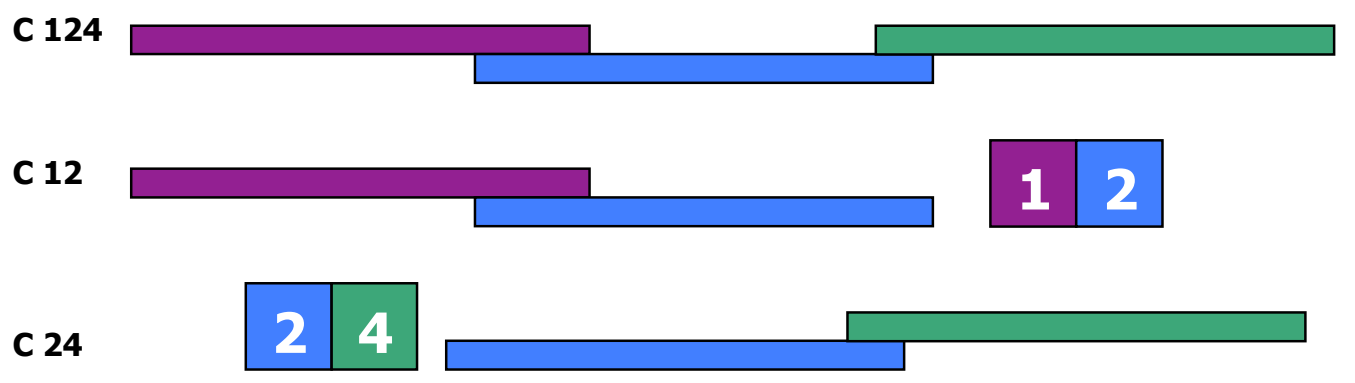

Figure 2. Assume that contig $\mathrm{c} 124$ can be formed with reads that are in groups 1,2 and 4 . Then in the first step of MUEGANO only contigs c12 and c24 will be formed. However in further steps, contig c124 will be recovered. 


\section{Proposition 2}

All contigs of exactly three reads will be present in at least one of the contigs resulting of the assembly of the first cycle (Fcontigs1) or at most in the second cycle of assembling (Fcontigs2).

Proof

Say that there are three reads, $x, y$ and $z$ for which $x$ and $y$ overlap and thus could form contig $c_{x y}$ and also $y$ and $z$ overlap, thus contig $c_{y z}$ could be formed but $x$ and $z$ do not overlap and so contig $c_{x z}$ does not exist (cannot be formed; see Figure 2).

If the three reads, $x, y$ and $z$ are all together in the same group $G_{m}$ in cycle one, then assembly $c_{x y z}$ will be formed in that cycle and will be present in Fcontigs1. The same occurs if the three reads are together in any of the $E_{m n}$ assemblies during the first cycle. But this is not warranted by the algorithm. However what is proved in Proposition 1 is that after the first cycle contigs $c_{x y}$ and $c_{y z}$ will be formed and they will be present in Fcontigs 1 and will proceed to cycle two as reads where, again, applying Proposition 1, they will form contigs $c_{x y z}$ that will be present in Fcontigs1. This line of reasoning can be generalized to prove that all contigs with any number of reads will be obtained in a finite number of steps (see Figure 2).

\section{Proposition 3}

All contigs existent in the original set of $\mathrm{N}$ reads are recovered by the MUEGANO algorithm.

Proof

Let $c$ be a contig with $r$ reads. Then the minimum number of independent overlaps in this contig must be $r-1$. Note that this contig will surly be formed if all the reads or sub-contigs forming it are feed in a single run to the assembler. Given that the procedure continues until either: a single run of the assembler is needed to process all reads (contigs) or until no new contigs are formed. In the first case, i.e., when all reads (contigs) are feed to the assembler the proposition is proved because this step must include at least all sub-contigs that form c. In the second case the contig c must be formed in a previous step.

\section{Notes}

Of course, if in any cycle no new contigs are formed the procedure stops because then there are not any overlapping reads. If the procedure continues it is because there were new contigs formed.

Also notice that there is no need to keep or process the singletons ${ }^{3}$ obtained in any of the steps; this is because if a singleton read did not found a pair to be assembled in a particular assembly, then, either it found a pair in other of the assemblies -and is already forming part of a contig, or it is a true singleton that cannot be assembled. A disadvantage of the procedure is that we lost count of the true singletons.

\footnotetext{
${ }^{3}$ A "singleton" is defined as a read that was not assembled in a particular assembly.
} 


\section{Applying MUEGANO to assemble maize reads}

In a maize project at Langebio we had more than 64 millions of reads, 63 of them in small reads of $100 \mathrm{bp}$ from 210 runs of the GS20-454 pyrosequencing apparatus, plus 555,000 reads of $250 \mathrm{pb}$ from three runs of the Flex-454 machine and around one million of Sanger reads with an average length of 800 bp, giving a total of more than 7,000 millions of bp of genomic DNA sequenced. No assembler program could cope with that quantity of reads in a single run given limitations in the RAM memory of the computers, thus there was a need to apply MUEGANO to this dataset.

For the assembly of the maize sequences we used two assemblers: the 454 assembler (version 1.0.53.17 of the the "off instrument software") and the 64bit version of the PCAP assembler (Huang, et. al., 2003). The 454 assembler does not allow parameter adjusting and the PCAP assembler was always run with the values recommended to tolerate highly repetitive overlaps, say: Min depth of coverage for repeats, $-I=75$ and Adjusted overlap score cutoff, $s=4500$ (Huang, X. and S. - P. Yang, 2005)

The assemblers where run in a cluster of 11 dual Xeon processors in which 10 nodes have $8 \mathrm{~Gb}$ of RAM and one node has $16 \mathrm{~Gb}$ of RAM. The maximum assembling capacity of the 454 assembler running on the cluster was calculated to be around 24 runs of 300,000 reads ( 7.2 millions of reads), while the maximum number of reads that the PCAP assembler was able to assemble was around 1.6 millions of reads. The details of the MUEGANO algorithm applied to this dataset are described in the following steps.

a) With the 213 runs of the GS20 and Flex machines we formed 18 sets with a maximum of 12 runs each (17 sets of 12 runs and one set of 9 runs). Given that the number of reads in each run of the 454 machines vary from 250,000 up to 500,000 reads (with a mean of around 300,000 reads) the sets were not formed at random but selecting runs with small and large number of reads to obtain the 18 groups, each one with around 3.6 millions of reads.

b) We did $18 \times 17 / 2=153$ runs of the 454 assembler with all possible pair combinations of the distinct sets. Each individual assembling comprised around 7,200,000 individual reads and produced around 55,000 contigs.

c) All contigs of at least $148 \mathrm{bp}$ obtained from the 153 individual assemblies $(8,028,352$ contigs) were feed to the NCBI BLASTCLUST (BLAST scorebased single-linkage clustering) program with parameters adjusted to cluster only identical sequences. The output of this procedure resulted in a non-redundant set of 4,821,833 contigs.

d) The set of non-redundant contigs was compared via blastn (Altschul, et al., 1997) to a dataset containing the reported sequences for the maize mitochondria and chloroplast. All sequences with a $95 \%$ or more identity with the maize organelles were set apart, obtaining a set of 4,681,391 non-redundant filtered contigs. The PHRED (Ewing, B. and P. 
Green,1998) quality of this set of contigs was reduced by a factor of 3 to allow flexibility in the assembly.

e) A run of PCAP with all 956,634 Sanger sequences produced a set of 100,407 contigs and 468,202 singletons after filtering by organelles.

f) The sets of sequences obtained in (d) and (e) where randomly allocated to seven sets of around 750,000 sequences, and all possible pair combinations ( $7 \times 6 / 2=21)$ of assemblies were performed using PCAP. These assemblies produced a total of 3,909,724 contigs that after filtering by redundancy (see " $c$ " above) were reduced to a set of 2,348,894 non-redundant contigs.

g) The set of non-redundant contigs obtained in step $f$ was divided in 3 groups of around 782,964 sequences and the assemblies with all possible pair combinations ( $3 \times 2 / 2=3$ ) were performed. This procedure produced a total of 702,514 contigs that after filtering by redundancy were reduced to a set of 606,187 non-redundant contigs.

Finally, the PCAP assembler was run with the 606,187 non-redundant contigs get in step $\mathrm{g}$, obtaining the set of final contigs.

\section{References}

Huang, X., J. Wang, et al. (2003). "PCAP: A Whole-Genome Assembly Program." Genome Res. 13(9): 2164-2170.

Huang, X. and S.-P. Yang (2005). Generating a Genome Assembly with PCAP. Current Protocols in Bioinformatics. A. D. Baxevanies, D. B. Davison, R. D. M. Pageet al. NY, John Wiley \& Sons, Inc. 2: 11.3.1-11.3.23.

Altschul, S. F., T. L. Madden, et al. (1997). "Gapped BLAST and PSI-BLAST: a new generation of protein database search programs." Nucl. Acids. Res. 25(17): 3389-3402.

Ewing, B. and P. Green (1998). "Base-Calling of Automated Sequencer Traces Using Phred. II. Error Probabilities." Genome Research 8: 185-194. 